\title{
Subcutaneous nodules of cysticercosis as a sign of asymptomatic neurocysticercosis in an HIV positive patient
}

\author{
Elisah Agaba ${ }^{[1]}$, Deepak Modi ${ }^{[1]}$, Ozge Gunduz ${ }^{[1]}$ and Zateen Modi ${ }^{[1]}$
}

[1]. Division of Dermatology, Faculty of Health Sciences, University of the Witwatersrand, Johannesburg, Gauteng, South Africa.

\begin{abstract}
Cysticercosis is caused by the hematogenous dissemination of the larval form (cysticercus) of Taenia solium. It can affect any organ or tissue in the body but commonly affects the subcutaneous tissue, central nervous system, eyes, and skeletal muscle. Skin lesions can assist as a marker in the diagnosis of asymptomatic neurocysticercosis in endemic areas. A 49-year-old HIV positive man presented with multiple cutaneous nodules confirmed as cysticercomas which led to the diagnosis of asymptomatic neurocysticercosis. He was successfully treated with albendazole and steroids at recommended doses with no adverse effects.
\end{abstract}

Keywords: Cysticercosis. Neurocysticercosis. HIV. Skin.

\section{INTRODUCTION}

Cysticercosis is a cestode infection caused by ingestion of Taenia solium eggs, usually shed in the stool of an asymptomatic human tapeworm carrier, and hematogenous dissemination of the larval form (cysticercus) of the pork tapeworm, T.solium. It is common in many countries in Central and South America, SubSaharan Africa, and Asia. However, its incidence is increasing in developed countries due to increased travel and migration from endemic areas ${ }^{1}$. The most common tissues affected are the subcutaneous tissue, central nervous system, eyes and skeletal muscle but any organ or tissue in the body can be affected ${ }^{2}$. Tissue cysticerci can remain dormant and asymptomatic for many years, but eventually, symptoms and signs develop following immunological inflammation.

We report the case of an HIV positive patient with asymptomatic neurocysticercosis whose diagnosis was made during the investigation of multiple subcutaneous nodules.

\section{CASE REPORT}

A 49-year-old man from Northern Namibia was referred to the Dermatology Clinic at a Private Hospital with a one-year history of asymptomatic but progressing subcutaneous nodules in June 2016. He had been on antiretroviral treatment (ART) for thirteen years for HIV infection diagnosed in 2003. He had been on a fixed dose combination treatment of tenofovir, emcitrabine and efavirenz since December 2015. His latest CD4 and viral

\footnotetext{
Corresponding author: Prof. Dr. Deepak Modi.

e-mail: profmodi@gmail.com

Received 11 May 2018

Accepted 18 July 2018
}

load levels indicated a good response to antiretroviral treatment (CD4 count, $370 \mathrm{cell} / \mathrm{mm}^{3}$ and viral load, $<40 \mathrm{copies} / \mathrm{ml}$ ). His hypertension was treated with amlodipine $10 \mathrm{mg} / \mathrm{day}$.

Clinical examination showed multiple, firm, mobile subcutaneous nodules with a diameter of 1 to $2 \mathrm{~cm}$ located on the trunk and upper extremities (Figure 1). Nodules varied from emerging dermal nodules to fibrotic hyperpigmented nodules. His physical and neurological examinations and fundoscopic examination of the eyes yielded normal findings. Ultrasound evaluation of the nodules showed hypoechoic cysts with mural nodules (Figure 2). Excisional biopsy of a nodule and hematoxylin and eosin staining demonstrated a cystic space with a thin fibrotic cyst wall surrounded by subcutaneous fatty tissue. Inside the cyst, eosinophils and a central intestinal cuticle of a transected parasitic infestation were seen. He had hypereosinophilia of $7.4 \%$ and a negative stool examination for parasites. Computed tomography (CT) scan of the brain showed bilateral multiple parenchymal nonenhancing cerebral cystic lesions with calcifications consistent with neurocysticercosis (Figure 3A).

The patient was diagnosed with disseminated cysticercosis and started on albendazole $800 \mathrm{mg} /$ day and prednisone 30 $\mathrm{mg} /$ day tapered over two weeks. No adverse reactions were reported. The skin lesions had disappeared both clinically and radiologically within a month. A repeat CT scan of the brain showed no active infection. Albendazole treatment was therefore stopped (Figure 3B). The patient continues receiving antiretroviral therapy without any sign of relapse or recurrence of the parasitic infection to date.

\section{DISCUSSION}

Cysticercosis is a public health problem especially in developing countries such as Namibia. Cysticercosis can affect almost any organ or tissue; however, the disseminated form 


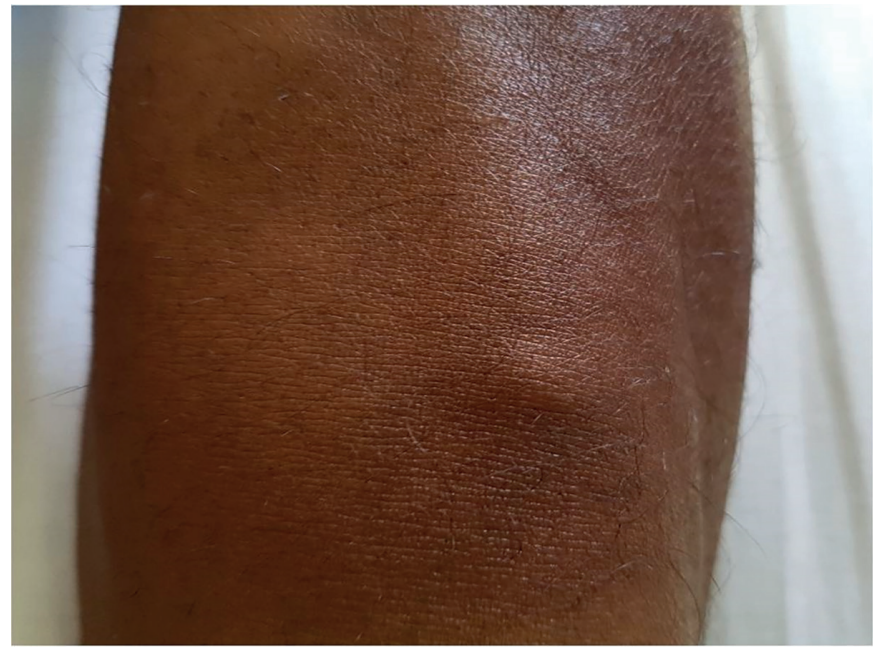

FIGURE 1: Subcutaneous nodule located on the right arm.

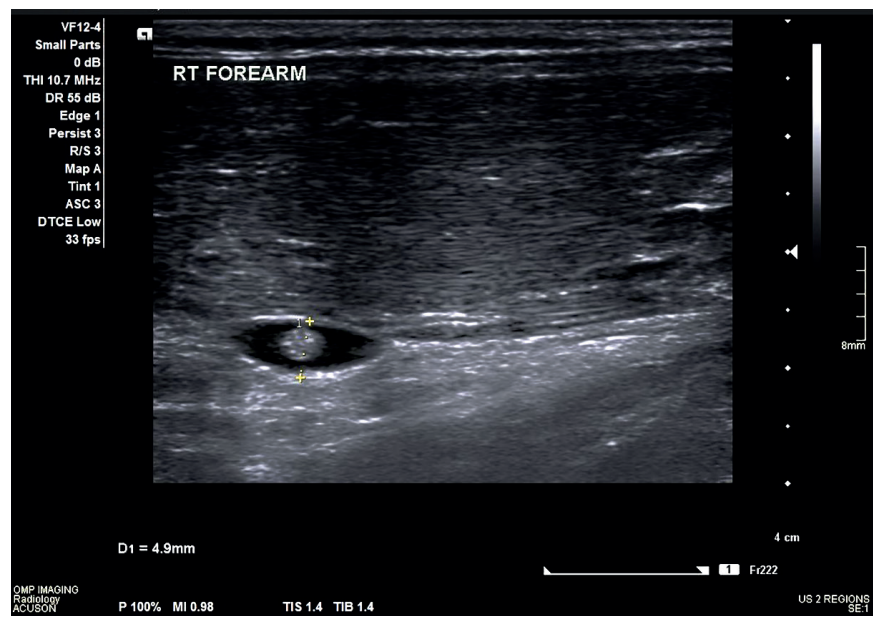

FIGURE 2: Ultrasound evaluation of the right forearm showed hypoechoic cyst with a mural nodule.

of cysticercosis is rarely reported ${ }^{2}$. Our patient did not show signs of eye involvement, but all other commonly affected organs (i.e., the subcutaneous tissue, central nervous system and skeletal muscle) were involved. Due to financial constraints screening was limited to commonly affected organs; therefore, the involvement of other organs could not be excluded.

The multiple tissue involvement of cysticercosis in our patient might be due to his HIV infection. In two studies from Northern Tanzania and Mozambique, although cysticercosis was fairly common among HIV positive patients, no relationship was found between seropositivity for $T$. solium and CD4 cell counts, treatment duration or type or disease stage ${ }^{3,4}$. There was also no difference in seropositivity for T. solium when comparing HIV positive and HIV negative patients. Symptoms of neurocysticercosis have been reported to start in HIV positive patients after commencement of highly active antiretroviral therapy suggestive of immune reconstitution inflammatory syndrome (IRIS) due to cysticercosis 5 .
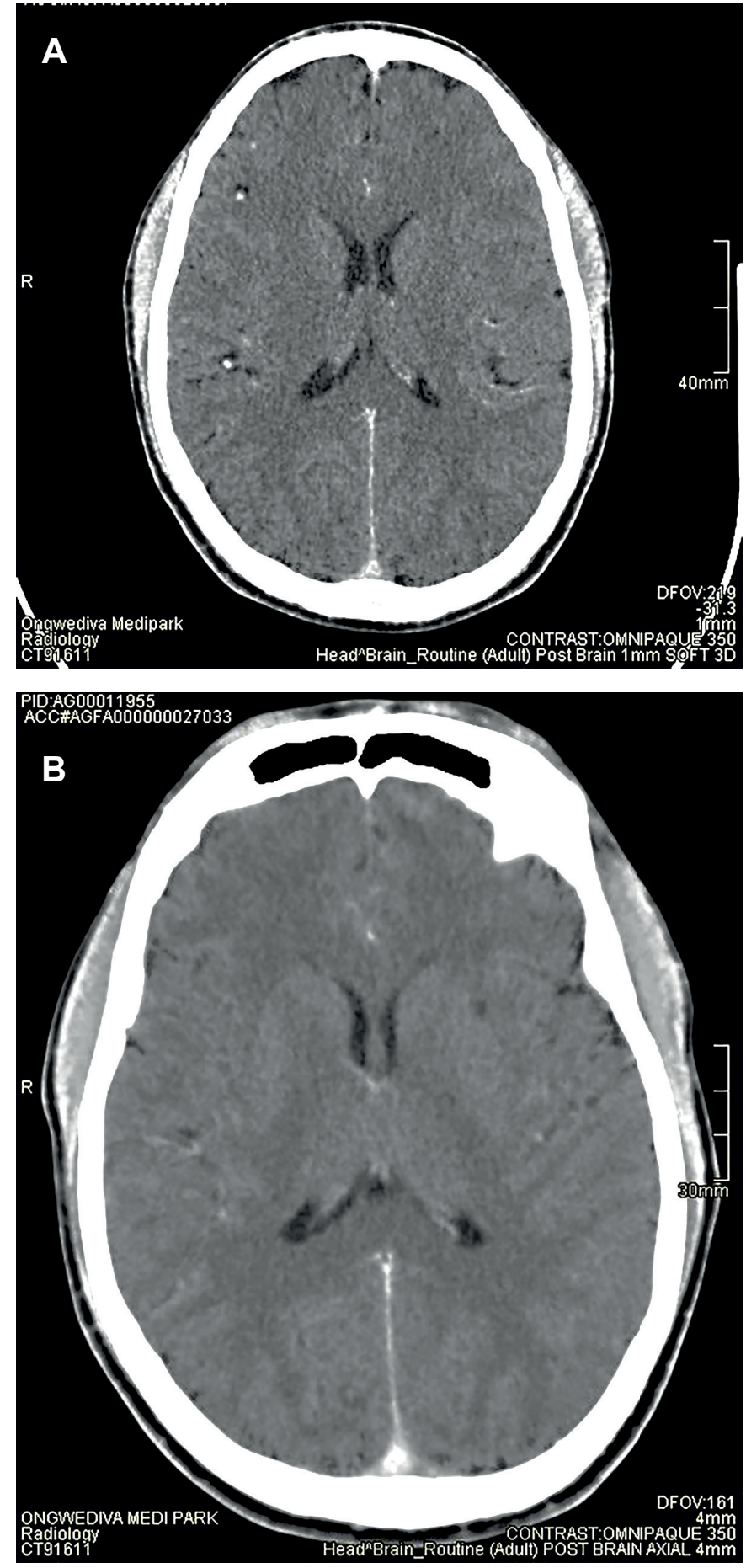

FIGURE 3: (A) Bilateral multiple parenchymal non-enhancing cerebral cystic lesions with calcifications, (B) No active infection on repeat CT scan of the brain.

Muscle or subcutaneous tissue involvement is the most common site of extraneural cysticercosis. Subcutaneous involvement occurs in about $54 \%$ of cases ${ }^{6}$. Cutaneous cysticercosis usually presents as multiple firm, elastic, round, mostly asymptomatic subcutaneous papulonodules most commonly on the trunk ${ }^{6}$. The diagnosis can be made by ultrasonic examination of the nodule in situ or histopathologic 
examination of an excised nodule which showed a cyst containing the cysticercus larvae. Cytological examination of fine-needle aspirates from nodules may reveal parasitic fragments. These nodules must be differentiated from other common skin tumors such as lipomas, epidermoid cysts, and fibromas. Infection of striated muscle is often asymptomatic but can cause discomfort if the nodules are inflamed. Intramuscular cysts often calcify and can thus be detected by radiographic examination?

Neurocysticercosis is the most common cause of seizures globally $\left(51 \%\right.$ of first onset seizures in Northern Namibia $\left.{ }^{8}\right)$ and may lead to a mortality rate of $>50 \%$ if left untreated. Other symptoms can include headache, nausea, and vomiting from elevated intracranial pressure, focal neurological deficits, psychological disturbances, and dementia. Neurocysticercosis can be asymptomatic in up to $50 \%$ of patients if there is a lack of host inflammatory response which is particularly compromised in advanced HIV infection making cysticercosis more likely to remain asymptomatic in these patients ${ }^{9}$.

In neurocysticercosis, antiparasitic drugs such as albendazole or praziquantel are effective ${ }^{9}$. The response rate to antihelminthic therapy in HIV patients with neurocysticercosis is $85 \%$ which is the same as patients who are HIV negative ${ }^{5}$. Steroid therapy is also used to prevent adverse inflammatory reactions and transient clinical deterioration caused by antihelminthic therapy. The risk of deterioration increases with a higher parasitic load.

We report a case of asymptomatic systemic neurocysticercosis in an HIV positive patient who presented with subcutaneous nodules only. Clinicians need to be alert to the possibility of disseminated cysticercosis infection in countries where there is endemicity.

\section{Conflict of Interest}

The authors declare that there is no conflict of interest.

\section{REFERENCES}

1. Garcia HH, Coyle CM, White AC Jr. Cysticercosis. In: Tropical Infectious Diseases: Principles, Pathogens and Practice. 3 rded, Guerrant RL, Walker DH, Weller PF (Eds), Saunders Elsevier, Philadelphia 2011. p.815.

2. Khandpur S, Kothiwala SK, Basnet B, Nangia R, Venkatesh HA, Sharma R. Extensive disseminated cysticercosis. Indian J Dermatol Venereol Leprol. 2014;80(2):137-40.

3. Schmidt V, Kositz C, Herbinger KH, Carabin H, Ngowi B, Naman E, et al. Association between Taenia solium infection and HIV/ AIDS in Northern Tanzania: a matched cross sectional-study. Infect Dis Poverty. 2016 1;5(1):111.

4. Noormahomed EV, Nhacupe N, Mascaro-Lazcano C, Mauaie MN, Buene T, Funzamo Ca, et al. A cross-sectional serological study of cysticercosis, schistosomiasis, toxocariasis and echinococcosis in HIV-a infected people in Beira, Mozambique. PLoS Negl Trop Dis. 2014;8(9):e3121.

5. Serpa JA, Moran A, Goodman JC, Giordano TP, White AC Jr. Neurocysticercosis in the HIV era: a case report and review of the literature. Am J Trop Med Hyg. 2007;77(1):113-7.

6. Schmidt DK, Jordaan HF, Schneider JW, Cilliers J. Cerebral and subcutaneous cysticercosis treated with albendazole. Int $\mathrm{J}$ Dermatol. 1995;34(8):574-9.

7. Jacob JC, Mathew NT. Pseudohypertrophic myopathy in cysticercosis. Neurology. 1968;18(8):767-71.

8. Gilman RH, delBrutto $\mathrm{OH}$, Garcia HH, Martinez M. Prevalence of taeniosis among patients with neurocysticercosis is related to severity of infection. The Cysticercosis Working Group in Peru. Neurology. 2000;55(7):1062.

9. Takayanagui OM, Jardim E. Therapy for neurocysticercosis: Comparison between albendazole and praziquantel. Arch Neurol. 1992;49(3):290-4. 